\title{
Infinity in Ontology and Mind
}

\author{
Nino B. Cocchiarella
}

\begin{abstract}
Two fundamental categories of any ontology are the category of objects and the category of universals. We discuss the question whether either of these categories can be infinite or not. In the category of objects, the subcategory of physical objects is examined within the context of different cosmological theories regarding the different kinds of fundamental objects in the universe. Abstract objects are discussed in terms of sets and the intensional objects of conceptual realism. The category of universals is discussed in terms of the three major theories of universals: nominalism, realism, and conceptualism. The finitude of mind pertains only to conceptualism. We consider the question of whether or not this finitude precludes impredicative concept formation. An explication of potential infinity, especially as applied to concepts and expressions, is given. We also briefly discuss a logic of plural objects, or groups of single objects (individuals), which is based on Bertrand Russell's (1903) notion of a class as many. The universal class as many does not exist in this logic if there are two or more single objects; but the issue is undecided if there is just one individual. We note that adding plural objects (groups) to an ontology with a countable infinity of individuals (single objects) does not generate an uncountable infinity of classes as many.
\end{abstract}

\section{Introduction}

Ontology, as originally described by Aristotle, is the study of being qua being, where being is not univocal but is divided into different categories. The two most fundamental categories are those of universals and objects, or things, respectively. Here, by a universal, and again we follow Aristotle, we mean that which can be predicated of things. ${ }^{1}$ Predication, of course, is what connects universals with objects. One important aspect of the role, or significance, of infinity in ontology, accordingly, is whether or not either of these categories, i.e., the category of objects or the category of universals, is, or can be, infinite. How infinity applies to mind in this regard is the question of whether or not there are, or can be, infinitely many concepts as intelligible universals, and whether or not the finitude of the human mind places limitations on the concepts that can be constructed.

\footnotetext{
${ }^{1}$ Aristotle, De Int. 17a39.
} 
Now the methodology of ontology, which, as we have said, is divided into different categories, is the analysis of those categories and the laws connecting them with one another, including in particular the nature of predication. The clearest and most precise way to analyze these categories is through the development of what is known as formal ontology, where the logico-grammatical forms and principles of a logistic system are formulated for the purpose of representing the different categories and the laws connecting them. ${ }^{2}$ A formal ontology in which ontological and logical categories are combined in a unified framework will then amount to a comprehensive deductive framework that is prior to all others in both logical and ontological structure. By proving the consistency of such a logistic system we can thereby show that the intuitive ontological framework associated with it is consistent as well.

One important role of infinity in ontology, accordingly, can then be understood as the determination of whether or not any of the categories of being, and in particular the categories of objects and universals, are, or can be, infinite as part of such a formal framework. In what follows we will consider some possible answers to this question.

\section{The Category of Objects}

We will consider the category of objects first in terms of the subcategory of physical objects and then the subcategory of abstract objects, such as classes, e.g., sets, or intensional objects (as the contents of concepts), neither of which have a predicative nature and therefore do not belong to the category of universals.

\subsection{Cosmology and the Objects of Nature}

On the level of objects we will first consider only the physical or otherwise natural objects that exist in nature. We assume that all macrophysical objects, including those of our commonsense framework and not just those in the cosmos at large, are ontologically grounded on the simplest and most fundamental objects of science, e.g., the elementary particles of quantum mechanics, or the vibrating strings of string theory. We assume in this regard that there can be an infinite number of physical objects only if there are an infinite number of elementary particles.

Because it is well-confirmed today we also assume the cosmological theory of the Big Bang, i.e., the so-called original "explosion" that occurred about 13.7 billion years ago and created the universe. We say "so-called" explosion because the Big Bang is perhaps better thought of as the simultaneous appearance of energy everywhere in the universe rather than an "explosion" that occurred at a single point in space. When the universe was created, the temperature was extremely hot, and the density of the material was enormous. According to the Wilkenson Microwave Anisotropy Probe (WMAP) satellite, the afterglow of the

${ }^{2}$ For a more detailed account of formal ontology Cocchiarella [2007], chapter 1. 
big bang is seen today as the background microwave radiation. ${ }^{3}$ As the latest data shows, 73 percent of the matter/energy of the universe is in the form of dark energy and 23 percent of the total matter/energy content is made up of dark matter, with only four percent being normal baryonic matter and energy, i.e., matter made up of such particles as neutrons and protons. ${ }^{4}$ Because we cannot speak of percentages of infinity, what this means is that the total matter/energy content of the universe is finite; and hence, because all of the physical objects that exist in nature are made up of this matter/energy content (and cannot be infinitesimally small), it follows that there can be only a finite (but very large) number of such objects.

The universe is still expanding as a result of the Big Bang, and apparently it will continue to expand until it reaches "heat death," or what is also called the "Big Freeze," when the temperature of the universe reaches near absolute zero. The big freeze is probably the final state of our universe, i.e., in effect it amounts to the end of the universe. Thus our universe has a beginning and will come to an end, which on this account means that time is finite. Also, because space and time are "quantized" in quantum physics, there is a smallest physically possible length - namely, the "Planck length" of $10^{-33} \mathrm{~cm}$. - and a smallest physically possible time, namely, the time it takes for light to cross the Planck length, which is $10^{-43}$ seconds. ${ }^{5}$ This means that space and time are discrete and not infinitely divisible. ${ }^{6}$

If space began when our universe began, then that would suggest that it too is finite. But as the WMAP has shown the curvature of space seems to be flat, which excludes a curved, bounded, and hence finite, space-time such as Einstein once favored. Of course the universe could be hyperspherical, and hence finite after all, but because it is so large it appears flat and Euclidean to us, just as a small part of the Earth's surface appears flat to us. ${ }^{7}$ But then, because the universe is accelerating in its expansion, it is said that it will continue to expand indefinitely, which means that space is potentially infinite even if at no time is it actually infinite. It is not clear, however, how the universe can continue to expand even after the end of time - unless of course time and space both exist before the Big Bang and after the Big Freeze. Such a sce-

\footnotetext{
${ }^{3}$ The WMAP satellite was a joint venture in 2001 of NASA and Princeton University. There are three experimental "proofs" of the big bang: the redshift of the galaxies, the cosmic back-ground microwave radiation, and nucleosynethsis of the elements. (See Greene [2004], pp. 429ff.)

${ }^{4}$ Dark energy is a force that acts in opposition to gravity and presently is causing the expansion of the universe. Dark matter, which makes up most of the mass in the universe, cannot be detected by any emitted radiation. Its existence is inferred by its gravitational effect. Determining the nature of both dark energy and dark matter is one of the most important problems in modern cosmology.

${ }^{5}$ The Planck length is the scale found at the big bang when the gravitational force was as strong as the other forces. See Gribbin [2001], p. 98, regarding the "quantization" of space and time. For more on this in the context of string theory, see Greene [2004], p.350.

${ }^{6}$ This does not mean that the mathematics of the continuum cannot be applied to space and time - just as the discreteness of the operations of a digital computer does not mean that we cannot solve calculus problems with computer programs.

${ }^{7}$ For different cosmological models of a finite universe see Luminet, et al [1999].
} 
nario seems in fact to be presupposed by several cosmological models, including the so-called many-worlds interpretation (MWI) of quantum mechanics and the recent string/M-theory, a superstring, supersymmetric theory about higherdimensional membranes. ${ }^{8}$

\subsection{The Multiverse of the Concordance Model}

Because of the expansion of the universe the most distant objects we can see now with the most powerful telescopes are about 40 billion lightyears $\left(4 \times 10^{26}\right.$ meters) away. ${ }^{9}$ A sphere of this radius, and hence our universe as well, is called a Hubble volume; and, according to some cosmologists, beyond the Hubble horizon there are other universes with the same laws of nature as ours, but with possibly different initial conditions. We are part of a multiverse, in other words, according to a view that is called the "concordance model". ${ }^{10}$ Space is assumed to be infinite on this model and because the distribution of matter is relatively uniform and represented by an ergodic random field it is also assumed that "there are infinitely many other regions the size of our observable universe, where every possible cosmic history is played out." ${ }^{11}$ Of course, as already noted, the multiverse could be hyperspherical, and hence finite after all, even though, because it is so large, it appears flat and Euclidean to us. Nevertheless, on this model, unlike the previous one, it is assumed both that space is infinite and that there are infinitely many physical objects in the multiverse; that is, even though there are only finitely many objects in each region, the total number of objects is infinite because the number of regions is infinite. This multiverse is essentially just the cosmos, however, because if the cosmic expansion were to decelerate then it would be physically possible to travel to regions beyond the Hubble horizon. On the other hand, if the acceleration of the universe's expansion continues indefinitely, then the rate of expansion will exceed the speed of light and the different regions will amount essentially to different possible worlds, all with the same laws of nature as ours. The objects in these regions can then be considered as physically possible objects, and quantifying over them would be different from quantifying over the "actual" objects of our region. The logic of a formal ontology representing this situation would be an S5 modal logic with actualist and possibilist quantifiers. ${ }^{12}$

\footnotetext{
${ }^{8} \mathrm{~A}$ fundamental property of string theory is that it is supersymmetric, incorporating not only the reference frame symmetries of relativity theory and the quantum-mechanical guage symmetries of the strong, weak, and electromagnetic forces, but also a supersymmetry principle that relates the properties of particles with a whole number of spin (bosons) with those with a half a whole (odd) number of spin (fermions). (See Greene [1999], chapter 9.)

${ }^{9}$ See Tegmark [2003]. Although the universe is only 13.7 billion years old and the light that is now reaching us from the most distant stars took that many years to reach us, those stars, because of the expansion of the universe, are now more than 13.7 billion lightyears away. They are in fact now about 40 billion lightyears away.

${ }^{10}$ Tegmark [2003].

${ }^{11}$ Ibid.

${ }^{12}$ Having all the same laws of nature the different regions would amount to an equivalence class of "possible worlds", which is a structure characteristic of S5 modal logic. For a formalization of the logic of actual and possible objects in S5 see Cocchiarella [2007], chapter
} 


\subsection{The Omnium of the MWI}

In Quantum mechanics ( $\mathbf{Q M})$ each particle in the universe is associated with a probability wave that specifies the different probabilities of where that particle might be located anywhere in the universe at each moment. Whether a particle is the same as its wave function, or whether the wave function is merely a mathematical construct that describes the particle's motion is one of the issues that distinguishes different versions of QM. In standard quantum mechanics, when a measurement is made and a particle is observed at a given location, then the probability of finding it at that location becomes 100 percent while the probability of finding it at any other location at that time drops to zero. This is what is meant in saying that the wave function "collapses". The many-worlds interpretation, (MWI), denies that a particle's wave function ever collapses.

Instead of a collapse of the wave function, what happens according to the MWI is that every potential outcome described in the particle's probability function is realized in a separate parallel world, so that anything that could happen in the sense of being physically possible according to $\mathbf{Q M}$ in fact does happen in some parallel world. ${ }^{13}$ All of the worlds accessible in this way from a given world when a measurement is made at a given moment have the same past up to that moment, but, except for the laws of nature, they differ thereafter in some way. An infinite number of parallel worlds populated by copies of ourselves is assumed in this way, where all of the worlds "co-exist" in a quantum superposition. ${ }^{14}$ Although the objects in those worlds are not "real" in the same sense in which the objects of our universe are real, nevertheless, they have an ontological status as objects of the multiverse, or what following Roger Penrose might preferably be called the omnium. ${ }^{15}$ This type of situation is represented in a formal ontology in terms of an $\mathbf{S} \mathbf{4}$ modal logic in which necessity and possibility are based on what is physically possible in QM. ${ }^{16}$ In other words, we distinguish between quantifying over the real objects of our universe from quantifying over the objects in other possible (parallel) worlds. There would then seem to be infinitely many possible objects, i.e., objects that exist in some possible world of the multiverse, even though in our universe there are only finitely many objects. ${ }^{17}$ branching into different parallel worlds takes place. Later proposals by Graham and DeWitt introduce the complicated notion of a measuring device that results in observations (by humans or automata) upon which the splitting into parallel worlds is based. See De Witt \& Graham [1973].

${ }^{14}$ Penrose [2004], p. 784.

${ }^{15}$ Penrose [2004], p.784.

${ }^{16}$ The modal logic is $\mathbf{S} \mathbf{4}$ because the accessibility relation between possible worlds is a partial ordering determined by the wavefunctions that split each universe into its related parallel universes. The result in effect is a branched-tree model of the universe something like what is described in McCall [1994].

${ }^{17}$ For a fuller account of MWI see De Witt \& Graham [1973].
} 


\subsection{String/M-Theory}

The situation is somewhat more complicated, and more speculative, in what is known as string/M-theory, where the goal is to unify general relativity and quantum mechanics. ${ }^{18}$ In the standard theory of $\mathbf{Q M}$ particles are zero-dimensional point-like objects with no internal structure, a situation that leads to problems at levels below the Planck scale where "quantum uncertainty becomes so violent that the smooth geometrical model of spacetime underlying general relativity is destroyed." ${ }^{19}$ General relativity and standard QM, in other words, are incompatible at levels below the Planck scale. In string theory, however, the particles of quantum mechanics are not zero-dimensional point-like objects but one-dimensional strings, the lengths of which are generally of the Planck length or longer, where the problem of quantum jitters can be overcome. In addition, one of the earliest results of the quantum mechanical equations of string theory was the existence of a string-particle, which like photons, has zero mass, but which, unlike photons, has spin-two, which is twice the spin of a photon. These were later found to be exactly the properties of a graviton, the messenger particle of the gravitational force. Gravity, in other words, is a fundamental part of string theory. Thus, not only can the incompatibility of QM with general relativity be resolved in string theory, but, in addition, string theory provides for a unification of QM with general relativity.

Now it is significant that the quantum mechanical equations of string theory do not work in four-dimensional spacetime but require ten or eleven dimensions instead. ${ }^{20}$ These extra dimensions are required because there are not enough vibrational patterns of strings in three-dimensional space to account for all of the particles of quantum mechanics. In fact, "there is an equation in string theory that demands that the number of independent vibrational patterns meet a very precise constraint; and how string theory determines the number of dimensions is a consequence of that constraint. ${ }^{21}$

Vibrating strings are the basic objects of string/M-theory, but, significantly, there are other "things" in this theory as well, namely, membranes, or what are simply called "branes", e.g., two- and three-dimensional branes, and, in general, $p$-branes, where $p$ is a positive integer less than 10. According to what

\footnotetext{
${ }^{18}$ The 'M' of "string/M-theory" is sometimes said to stand for 'Membrane theory', sometimes for 'Matrix theory', and sometimes even for 'Mystery theory' or 'Mother of all string theories'.

${ }^{19}$ Greene [2004], p. 349. As Greene notes, "the point-particle description is merely an idealization and ... in the real world elementary particles do have some spatial extent" (p.157). Some scientists have tried work with "blobs" or "nuggets", but, because of quantummechanical probability and the impossibility of faster-than-light speed transmission of information, it was found to be too difficult to construct a QM theory that works with such objects (Ibid., p. 158).

${ }^{20}$ See Greene [2004], chapter 12 for a fuller discussion of these points,

${ }^{21}$ Ibid., pp. 370f. Originally, there were five competing string theories each with six extra spatial dimensions; but then, in 1995, the physicist Edward Witten showed that from the point of view of another seventh dimension all five theories are equivalent and can be translated into one another. The result is eleven dimensions in all, counting time as well. (For more on this see Greene [2004], chapter 13.)
} 
is called "the braneworld scenario," the universe is a three-brane. In fact, our three-brane world might not be anything special; that is, "there could be many other branes, of various dimensions, floating within string/M-theory's higher dimensional expanse." ${ }^{22}$ It is not clear how many strings there are, but because both particles and their properties are explained in terms of the vibrational patterns of strings there would seem to be at least a potential infinity of both. Also, because gravitons can move from one brane world to another it is not clear how many gravitons there can be.

There is a new theory, incidentally, that assumes another three-brane universe no more than a millimeter away from ours, the space between us being another fourth dimension. This is the cyclic (or "Big Splat") model that Paul Steinhardt, the Einstein Professor of Science at Princeton University, and Neil Turok of Cambridge University have recently developed as an alternative to the inflationary theory about what happened after the Big Bang. We have no evidence of this other three-dimensional universe or the objects in it because, according to string theory, the endpoints of the open strings that make up the matter that we are made of are "stuck" to our 3-space, which makes it impossible for us to experience other dimensions in hyperspace. Gravitons, however, are closed strings and can move from one space to another, which explains the weakness of the gravitational force compared to the others.

On the cyclic model, our universe and the other three-brane universe near us are attracted to one another, and this attraction is part of what drives the cosmological evolution of both universes. At one stage of this evolution, the two branes collide (the big splat), and then rebound and begin to expand. The energy generated by the collision is tremendous and results in high-temperature radiation and matter in each universe with a profile that is nearly identical to what is produced in the inflationary model. But instead of all of space being infinitely compressed at the beginning, as on the inflationary theory, only the dimension between the two universes is compressed. Then, as a result of "dark energy", which, unlike matter and radiation, is gravitationally self-repulsive, the universes begin to expand. ${ }^{23}$ In this model, unlike the inflationary theory, the universe goes directly to a radiation-dominated period after the rebound, and then to a matter-dominated period in which atoms and galaxies and larger scale structures form, and then, after about fourteen billion years, to a phase dominated by dark energy in which the expansion accelerates. We are at present living in a time when the universe is expanding at an accelerating rate, which means that over time (about a trillion years from the present on the cyclic model) the distribution of matter and radiation in the universe will thin out, driving the universe into what is essentially a vacuum state. After that the attraction between the two universes will be sufficient to bring them together again for another collision. Thus, "the branes engage in an endless cycle of collision, rebound, and collision once again, eternally regenerating their expanding three-

\footnotetext{
${ }^{22}$ Greene [2004], p. 412.

${ }^{23}$ A finite expansion of an infinite space results only in an infinite space, just as adding a finite cardinal number to an infinite cardinal number results in that infinite cardinal number.
} 
dimensional worlds." 24

In the inflationary theory the universe has a beginning, the Big Bang, and an end (assuming the accelerating expansion continues), namely, the Big Freeze. On the cyclic model, the universe goes through an endless cycle of collision, expansion and rebound. Space on this model is infinite throughout the evolution of the universe, and time is endless, and presumably the total number of objects that exist in one cycle or another is infinite - though it would seem that only the finitely many objects that exist in our cycle are "actual," or "real," whereas those that exist in other cycles are like the possible objects of parallel worlds. In a formal ontology this situation would be described by an S5 modal logic with actualist and possibilist quantifiers. ${ }^{25}$

We should note, however, that the cyclic theory has a problem with the buildup of entropy in each cycle, which adds to the entropy of earlier cycles. ${ }^{26}$ The problem is that if entropy increases, then, according to general relativity theory, the duration of each cycle increases as well; that is, each successive cycle lasts longer than its predecessors. Earlier cycles would then be shorter and shorter, returning us in a finite amount of time to a beginning after all.

We have assumed throughout this discussion that all macrophysical objects are grounded in the fundamental particles of quantum mechanics, or the strings of string/M-theory - even if some of these objects are emergent and not reducible to the fundamental particles or strings - and hence that their number does not change the number of objects in ontology in a critical way.

We conclude, accordingly, that even if there are only finitely many elementary particles, and hence physical objects, in the universe, i.e., in our Hubble region of spacetime, there are nevertheless infinitely many objects that our possibilist quantifiers should range over.

\subsection{Summary of the Cardinality of the Category of Phys- ical Objects}

No matter which cosmological model we adopt, the matter and energy, both dark and otherwise, within our universe -i.e., within the Hubble volume of our system of galaxies - is finite, and hence the number of objects, both elementary and composite, that can be ontologically grounded on that matter and energy is also finite. Time and space have a beginning with the Big Bang on the standard model, and time, even if not also space, has an end (of sorts) with the Big Freeze.

The number of objects in the multiverse of the concordance model, and in that sense the number of physically possible objects in our universe, is said to be infinite - but only because, on this model, space and the number of Hubble volumes in space is assumed to be infinite. If the universe is hyperspherical and

\footnotetext{
${ }^{24}$ Greene [2004], p. 407.

${ }^{25}$ Here the equivalence class of possible worlds is the class of universes that exist at some cycle or other. We can include the objects in the other three-brane world here as well. A separate issue is how the two three-brane worlds are themselves treated. That is, are they included in the domain of objects as well?

${ }^{26}$ See Greene ([2004], p. 410.
} 
only appears flat and Euclidean because it is so large, however, then space is not infinite but finite, and therefore the number of Hubble volumes, or parallel worlds, is finite as well, in which case, because each such volume has only finitely many physical objects, the number of physically possible objects is also finite. Of course, because the universe is accelerating in its expansion, it will continue to expand indefinitely, which means that space is potentially infinite even if at no time is it actually infinite. It is not clear, however, that this also means that the number of parallel worlds, and therefore physical objects, is also potentially infinite as well.

In the many-worlds model space is also assumed to be infinite - though again that assumption seems to be gratuitous, based as it is on the apparent flatness of our world. The number of objects in the omnium of the MWI, and in that sense the number of physically possible objects in our world, is also assumed to be infinite - but only because the number of parallel worlds in the omnium is assumed to be infinite. If the universe is not flat but hyperspherical, then because space and time are quantized, and hence discrete, there are only a finite number of "moments" at which a split in the universe can occur, and therefore there would only be a finite number of parallel worlds, each having in turn only a finite number splits into new parallel worlds, which means that the total number of worlds and physical objects in them is also finite.

In the cyclic model of string/M-theory both space and time are assumed to be infinite, and although the number of objects in each 3-D membrane that repeatedly collide with each other are finite, nevertheless the number of objects that exist in those membranes in some cycle or other, and in that sense the number of physically possible objects, is assumed to be infinite - but only because the number of cycles is assumed to be infinite. If there is a beginning to those cycles, then again there are only a finite number of cycles so far, and therefore a finite number of physically possible objects so far as well - though potentially those cycles can continue on indefinitely, and therefore the number of physically possible objects is potentially infinite, even if finite at any given cycle.

There are other cosmological models as well that we have not considered here, but the above models are the better known and most widely discussed today. Our general conclusion with respect to these models is that the number of physical objects in our universe is finite, but that the number of physically possible objects is infinite - but only because of an assumption that space, and in at least one case time as well, is infinite.

\section{Abstract Objects: Set Theory}

We now turn briefly to abstract objects, in particular the classes of set theory, which, despite sometimes being called universals, ${ }^{27}$ do not have a predicative nature and therefore are not universals. Later, we will also consider intensional objects as the contents of predicable concepts, but which as hypostatized objects

${ }^{27}$ See, e.g., Quine [1953], p.121. 
also do not have a predicative nature and hence also do not belong to the category of universals.

In set theory, and in Zermelo-Fränkel set theory $(\mathbf{Z F})$ in particular, our informal notion of a class is replaced by the iterative concept of a set. On this concept, sets are formed in stages by iterating Cantor's power-set operation: $X \rightarrow \mathcal{P}(X)$, where $\mathcal{P}(X)$ is the power-set of $X$, i.e., the set of all subsets of $X$. It is because of their formation in this manner that sets are sometimes said to be classes that have their being in their members, as opposed, e.g., to classes that have their being in the concepts whose extensions they are. The empty set has no members, however, and therefore cannot be said to have its being in its members. It is perhaps questionable then of how pure sets, which are built up from the empty set by the power-set operation, can really be said to have their being in their members.

Set theory, as is well-known, provides a foundation for mathematics, including in particular an important and useful explication of infinity. A set is infinite, of course, if, and only if, it is not finite, where by finite we mean that the set has finitely many members - i.e., that for some natural number $n$, the set has $n$ many members. But a non-numerical definition-i.e., a definition that does not assume the concept of a natural number - can also be given. In 1888, for example, Dedekind suggested that a class is finite if, and only if, it is not equipollent-i.e., equal in power- to any of its proper subclasses. ${ }^{28}$ Significantly, although every class that is finite in the ordinary sense is Dedekind finite, the converse can be shown to hold only if we assume the axiom of choice. Other non-numerical definitions have also been given. ${ }^{29}$ We will not go into those definitions here, but we will assume the ordinary, or commonsense, notion instead.

Now one of the things that Cantor showed is that the power-set of any set always has more members than that set; that is, $X \prec \mathcal{P}(X) .{ }^{30}$ This means that the cardinal number of the set of natural numbers, $\aleph_{0}$ (Aleph-null), is less than that of its power-set, which has cardinality $2^{\aleph_{0}}$, i.e., $\aleph_{0} \prec 2^{\aleph_{0}}$. But then $\mathcal{P}(X)$ has fewer members than its power-set $\mathcal{P} \mathcal{P}(X)$, i.e., $2^{\aleph_{0}} \prec 2^{2^{\aleph_{0}}}$, which in turn has fewer members than its power-set, $\mathcal{P} \mathcal{P} \mathcal{P}(X)$, and so on ad infinitum. There is no end, in other words, to the hierarchy of infinite sets, and therefore no universal set.

Now although set theory is a powerful mathematical framework within which all of mathematics can be developed, it is not clear that any of the higher reaches of set theory beyond $2^{\aleph_{0}}$ has any application to the natural world. This is troubling ontologically because pure sets, i.e., sets built up exclusively from the empty set, exist independently of the natural world, if in fact they really exist at all. But if they are assumed to exist in a given formal ontology, then they must be included among the values of the objectual variables, in which

\footnotetext{
${ }^{28}$ Dedekind [1988]. Classes are equipollent if they can be put into a one-to-one correspondence with each other.

${ }^{29}$ A systematic survey of definitions can be found in Tarski [1924].

${ }^{30}$ In set theory, $x \preceq y$ just in case there is a subset $z$ of $y$, i.e., $z \subseteq y$, such that $x$ is equipollent to $z$. Then, $x \prec y$ if $x \preceq y$ but $y \npreceq x$.
} 
case that ontology is committed to an endless hierarchy of infinite sets, most of which are irrelevant to the natural world.

Set theory does provide a useful model-theoretic framework for formal ontology. That is, even though membership is not at all the same as predication, different theories of predication can be modeled in terms of membership or functions constructed in terms of membership. This is convenient because it allows us to apply the set-theoretic notions of finitude and infinity when constructing models of a given formal ontology. Nevertheless, it is one thing to use set theory as a modeling tool in formal ontology, but quite another to adopt set theory as a ontology in its own right.

\section{The category of Universals}

An ontology consists not only of the category of objects, physical or abstract, but also of the category of universals. Having discussed the category of objects we turn now to the category of universals. A universal, it will be remembered, is what is predicated of things, i.e., of objects.

What then are universals? Traditionally, there have been three main theories of universals: nominalism, conceptualism, and realism.

The difference between these three types of theories depends on the universals that each takes to be predicable of things. Nominalism, for example, maintains that only the tokens of the predicate expressions of a language can be predicated of things, whereas conceptualism maintains that such tokens of predicates can be true or false of things only because they stand for predicable concepts, where by a predicable concept we mean a cognitive capacity (intelligible universal) that underlies predication in thought and our rule-following ability in the use of a predicate expression of natural language. Realism, on the other hand, maintains that there are real universals, namely, properties and relations, that are the basis of predication in reality. In logical realism, which is a modern form of Platonism, properties and relations, and perhaps even propositions, exist independently of whether or not they are realized in nature, and even of whether or not there is a natural world at all. In natural realism, however, (natural) properties and relations exist only as part nature's causal matrix, which means that they do not exist independently of that causal structure, and that they are in principle realizable in nature as well.

\subsection{Nominalism}

We begin with nominalism, which maintains that only the tokens of predicate expressions of a language can be predicated of things, and that predication is to be understood simply as a predicate's being true, or false, of things. Predicate quantifiers can be allowed in a formal ontology for nominalism, but only

under a substitutional interpretation, and where only the first-order formulas 
of an applied (formal) language can be substituted for predicate variables. ${ }^{31}$ This means that the underlying logic for nominalism is standard second-order "predicative" logic.

Traditionally, nominalism also maintained that there are no abstract entities of any type, and hence that there are no abstract objects as well as no abstract universals. This means that only tokens of predicate expressions can be predicated of things, because otherwise one must assume abstract types of predicate expressions, or classes of similar tokens, which presumably would be abstract entities. ${ }^{32}$ The token expressions are generated in speech acts and in writing by us, of course, which means that there can be at most a finite number of such tokens. It also means, as Leon Henkin has observed, that a nominalistic interpretation of the sentences of a language adequate for most mathematical discourse is not possible. ${ }^{33}$ The situation is different, however, if infinitely many tokens are allowed. Of course, one might claim that even if there are only a finite number of tokens at any given time, nevertheless that number can be increased indefinitely, which suggest that there can be a potential infinity of tokens even if not an actual infinity. The notion of a potential, as opposed to an actual, infinity is somewhat problematic, however. But it is a notion we will later return to, and explicate, in our discussion of conceptualism.

Some nominalists do allow for abstract particulars as features, or parts, of things, which they sometimes call tropes. ${ }^{34}$ It is even claimed that Aristotle was committed to such particulars, as when he says that whiteness is "in" Socrates..$^{35}$ But then some of these philosophers also claim that ordinary objects in the world are just sums of tropes, ${ }^{36}$ which is certainly not Aristotle's position. Because they do not exist otherwise than as parts, or features, of ordinary objects in the world, it does not seem that there can be any more of these tropes than there are such objects to begin with.

\subsection{Logical Realism}

There are two types of realism, as we have noted, namely, logical realism, which is a modern form of Platonism, and natural realism, which, if supplemented with a logic of natural kinds, can be developed as a modern modal form of Aristotle's moderate realism. ${ }^{37}$ The two differ in a number of respects. In logical realism, for example, properties and relations - and perhaps propositions as well - exist as abstract entities independently of the world and even of whether or not

\footnotetext{
${ }^{31}$ Strictly speaking, formulas with free predicate variables can also be allowed as substituends. What is excluded are formulas with bound predicate variables. See Cocchiarella [2007], chapter 4, for a fuller account of nominalism as a formal theory of predication.

${ }^{32} \mathrm{As}$ an alternative to classes, e.g., sets, as abstract entities, the nominalist could adopt "classes as many" as plural objects, which we describe below under conceptualism.

${ }^{33}$ Henkin [1962].

${ }^{34}$ See Campbell [1981] for an account of tropism as an ontology.

${ }^{35} \mathrm{It}$ is perhaps inappropriate to refer to these particulars as abstract objects, but that is the standard terminology today.

${ }^{36}$ See, e.g., Williams [1953] for such a position.

${ }^{37}$ See Cocchiarella [2007], chapters 4 and 12 for accounts of logical and natural realism, respectively, as formal ontologies.
} 
there is a world. The properties and relations of natural realism, on the other hand, are part of nature's causal matrix and do not exist independently of that structure. A full "impredicative" comprehension principle - i.e., a principle that validates the existence of properties and relations corresponding to complex formulas containing predicate quantifiers - is valid in the formal ontology of logical realism, which does not mean that there are complex properties and relations, e.g., that logical relations are components of such universals. Rather, what the instances of such a principle represent are the logical relations that obtain between properties and relations that have their being in the Platonic network of logical space. Complex formulas do not in general represent natural properties and relations, on the other hand, and particular cases can be assumed to do so only on the basis of empirical investigations. Natural properties and relations, like those of some transuranic elements, might have no instances in our universe, but because they exist as part of nature's causal matrix they must, as a matter of a natural necessity, have instances in some possible world in the multiverse.

Some logically real properties and relations, on the other hand, cannot, on logical grounds alone, have instances. It is logically impossible, for example, that there be a round square. ${ }^{38}$ But unlike sets, which have their being in their members, a property or relation does not have its being in its instances. Thus, unlike the problem of the empty set, which, like sets in general, is said to have its being in its members, there is no problem in logical realism with a necessarily vacuous property such as being a round square. The difference between sets and logically real properties and relations is also indicated by the fact that the hierarchy of sets, as indicated by Cantor's power-set operation, is built from the bottom up without end, which means that there can be no universal set. Logically real properties are not built from the bottom up, and the property of being self-identical is a universal property that is shared by every object whatsoever. This shows that sets in the sense of the iterative concept are not the extensions of logically real properties and relations, and that an infinity of the latter is not like the unending infinities of the former.

The extensions of logically real properties are classes in the logical sense, i.e., classes that have their being in the property or concept whose extensions they are. The "sets" of the so-called set theory NFU (New Foundations with Urelements) are really classes in this sense. NFU has a universal class, and its classes are not built up in terms of Cantor's power-set operation, which fails when applied to the universal class. In fact, NFU is equiconsistent with simple type theory and also with the system $\boldsymbol{\lambda} \mathbf{H S} \mathbf{T}^{*}$, which is a second-order predicate logic with nominalized predicates as abstract singular terms, and which can be taken as a reconstruction of Bertrand Russell's early 1903 form of logical realism. ${ }^{39}$ Because the values of the predicate variables can be mapped into the

\footnotetext{
${ }^{38}$ Because the logical truths of first- and higher-order logic are not recursive, we cannot have an effective rule that would exclude properties and relations that logically cannot have instances.

${ }^{39}$ See Cocchiarella [1987], chapters 2 and 4 and [2007], chapters 4 and 5, for a fuller account of these relationships.
} 
values of the objectual variables in this logic, we need not assume that there are any more than $\aleph_{0}$ many logically real properties and relations, or at most $2^{\aleph_{0}}$ many such properties if we assume that the domain of objects has $\aleph_{0}$ many members.

\subsection{Natural Realism}

The situation is more difficult to determine in natural realism where we might assume a difference between natural kinds of things and the natural properties of those kinds of things. Natural kinds are stable causal structures, or mechanisms, in nature that are the bases of the powers or capacities to act, behave, function, etc., in certain determinate ways that objects belonging to those natural kinds have. Natural properties and relations are then the physical states or conditions of the objects having those natural kinds of causal structures. ${ }^{40}$ In the standard model of quantum mechanics, for example, we have a veritable zoo of about 200 different natural kinds of particles. At the fundamental level of the elementary particles of matter, i.e., simple, structureless, noncomposite particles of matter, we have three families of six leptons - namely, the electron, the muon, and the tau particles, each with their respective neutrino particles - and three families of six quarks (sometimes described as "flavors") — namely Up and Down, Charm and Strange, and Top and Bottom. Composite particles are made of quarks and leptons. Hadrons, for example, is a family of ("bulky") particles composed of quarks. There are two subfamilies of hadrons, namely the baryons, each of which is made of three quarks, and the mesons, each of which is made of one quark and one antiquark. Protons, for example, are baryons made of two Up quarks and one Down quark, and neutrons are baryons made of two Down quarks and one Up quark.

In addition to these different families and subfamilies of particles of matter, there are also four kinds of messenger particles, i.e., particles that carry one of the four fundamental forces, namely, the photons for the electromagnetic force, the gluons for the strong nuclear force, the $W$ and $Z$ gauge boson particles for the weak nuclear force, and the gravitons for the gravitational force. All of these and many other types of particles might well be described as natural kinds of particles, each having a different function and each made of a different kind of "stuff" that explains their different properties, e.g., such properties as mass, electric charge, spin (angular momentum), etc. All together, there would seem to be a finite number of natural kinds of particles and a finite number of properties that those particles are described as having in the standard model of quantum mechanics.

The situation is quite different in string theory where all matter and all force particles are composed of the same kind of "stuff", namely vibrating strings. Each elementary particle, for example, is a single string, and all strings are identical in their nature as strings. The differences between the particles are explained in this theory in terms of the different resonant vibrational patterns that

\footnotetext{
${ }^{40}$ See Cocchiarella [2007], chapter 12, for a description of a formal ontology based on this distinction between natural kinds and natural properties.
} 
can be executed by strings. ${ }^{41}$ That is, both the particles and their properties are explained in terms of the different vibrational patterns of strings, and of course there are an infinite number of vibrational patterns that strings can execute. ${ }^{42}$ There can be, in other words, not only an infinite number of strings, but also an infinite number of natural properties and relations that can be grounded in the vibrational patterns of strings - even though there are only a finite number of strings and vibrational patterns actually realized in our universe.

There are many other stable causal structures on higher-levels of ontological analysis, of course, such as the different natural kinds of atoms, or, on an even higher level of complexity, the natural kinds of plants and animals. The structures and the laws of nature regarding chemical elements, i.e., atoms, and the complex molecules built up from them, including much of organic chemistry, may very likely be reducible to the particles of quantum mechanics, and perhaps this is true even of some elementary forms of life. It has been doubted, however, that the same can be said for vertebrates once information theory and the information contained in the genome of vertebrates is taken into consideration.

\subsection{Emergent Natural Kinds}

Both reductionists and anti-reductionists agree that a living system is an information processing system, i.e., a structural hierarchy of functioning units that has acquired through evolution the ability to store and process the information necessary for its own accurate reproduction. ${ }^{43}$ DNA stores the hereditary information from which the entire organism is derived, and it does this in terms of a linear sequence of "symbols", namely, the nucleic acid bases $\mathbf{A}$ (denine), $\mathbf{T}$ (hymine), $\mathbf{C}$ (ytosine) and $\mathbf{G}$ (uanine), which can be regarded as the "letters of a language" with informational content. Transmitting information amounts to a form of knowledge, factual or conceptual, by means of a linear sequence of symbols ordered according to the constants of such a "language". Here the base sequence of DNA is the encoded message at the source of the living channel and the amino acid sequence of proteins is the message that is finally received. Natural selection acts, under all current thought, through the sequence of amino acids in proteins.

Information has been developed as a scientific theory ever since Claude Shannon's work in the 1940s. ${ }^{44}$ One part of this development is the idea that whereas entropy, as a measure of disorder, is the basic random element in the universe, information, as a measure of order, is the basic nonrandom element. Information, in other words, can be viewed as a principle that can "inform" the material world in the way that DNA instructs the machinery of cells to build an organism. What Shannon proved was that information can be sent free from error even under noisy conditions in a communication system (such as the genome) so long as it is coded in the proper way. Shannon's second theorem in effect states

\footnotetext{
${ }^{41}$ Greene [1999], p. 146.

${ }^{42}$ Ibid., p. 151.

${ }^{43}$ Mutations, of course, are changes in the informational content of the hereditary units.

${ }^{44}$ See Shannon adn Weaver [1949].
} 
that an ideal code always exists that gives as much accuracy as we like within the constraints of a channel's capacity. For living systems the claim is that this allows for what the biophysicist Lila Gatlin calls "second-theorem evolution," which, according to Gatlin, is fundamental to understanding the evolution of vertebrates.

Thus, according to Gatlin, Shannon's second theorem of information theory shows that "it is possible within limited ranges of the entropy variables to increase the fidelity of a message without loss of potential message variety, provided that the entropy variables change in just the proper way ... [and that] this is the specific strategy which makes vertebrates master players relative to the lower organisms...." 45 Second-theorem selection and Darwinian selection, Gatlin says,

might sometimes conflict. A change in the base sequence of DNA might lower transmission error but produce a protein less fit for its environment, or conversely. ... Darwinian selection could also act to reduce transmission error in the channel. This would be equivalent to improving the efficiency of a machine by improving its hardware. However, the second law of thermodynamics quickly places an upper bound on improved efficiency by this method. ... If improving the hardware of the computer were the only method of improving the efficiency of information processing in the living system, evolution would have reached it apex in E. coli. ${ }^{46}$

The information contained in the genome of vertebrates, in other words, cannot be accounted for in terms of Darwinian evolution alone, according to Gatlin, but requires second-law evolution as well.

It is not clear what the limits are for the maximum number of natural kinds, i.e., stable causal structures, living or otherwise, that are possible in nature over and above the fundamental level of particles in quantum mechanics or the strings of string theory. And just as it is not clear to what extent these structures are reducible in principle to the fundamental level of particles or strings, so too it is not clear to what extent the natural properties and relations that can obtain between these higher-level structures can be reduced to the properties of particles or strings.

\subsection{Conceptualism and the Finitary Mind}

Predication in conceptualism of the type we will consider here is based on the exercise and mutual saturation of two types of concepts as cognitive capacities, namely predicable concepts and referential concepts. ${ }^{47}$ These capacities are the cognitive structures that underlie our rule following abilities in the use of the

\footnotetext{
${ }^{45}$ Gatlin [1972], p.196.

${ }^{46}$ Ibid., p.202.

${ }^{47}$ See Cocchiarella [2007], chapter 4, for a fuller discussion of conceptualism as a formal ontology.
} 
predicate and referential expressions of language. Referential concepts, which are what noun phrases in natural language stand for, are represented in formal ontology by quantifier phrases applied to names, both proper and common and complex or simple. Predicable concepts are of course represented by predicate phrases, both complex and simple.

There are two major stages of concept formation with respect to predicable concepts, namely, an initial stage at which only so-called "predicative" concepts are formed, i.e., concepts that do not presuppose a totality to which they belong, and a second later stage, usually occurring only in post-adolescence, at which socalled "impredicative" concepts can be formed. ${ }^{48}$ This is because impredicative concepts - such as those of a least upper bound of a set of real numbers, or the limit of a converging sequence of real numbers-presuppose a totality to which they belong and are said to violate a vicious-circle principle. The implicit claim is that, given the finitary structure and limitations of the central nervous system, impredicative concept formation is biologically impossible - at least as far as concepts specified in terms of infinite totalities are concerned. ${ }^{49}$ On this argument, the only justification that we can give for the use of impredicative expressions in our formal theory is the Platonist assumption that there are logically real properties or classes that are the intensions or extensions of such expressions. ${ }^{50}$

The representation in conceptualism of a logic of strictly "predicative" concepts, i.e., concepts that do not violate the vicious-circle principle, is somewhat different from the standard "predicative" logic for nominalism. In particular, the conceptualist logic is "free" of existential presuppositions regarding predicate expressions, i.e., free of assuming that they stand for "predicative" concepts, just as proper names might fail to name anything. In addition, the comprehension principle represents a pattern of reflective abstraction that conceptually drives the logic into a ramified hierarchy in the attempt to close the gap between predicate expressions that stand for concepts and those that do not. ${ }^{51}$ Of course, if one thinks of the ramified hierarchy as representing logically real properties or classes, as the Platonist does, then the idea that there is a "progressive creation" of properties or classes in this hierarchy is indeed at best a metaphor as the Platonist claims. ${ }^{52}$ But in conceptualism the hierarchy is viewed as a progressive construction of concepts as cognitive capacities, and the drive for closure is strictly a conceptual matter, and not one of a "progressive creation" of properties or classes as abstract entities.

In the second major stage of concept formation impredicative concept formation becomes possible on the basis of an idealized, as opposed to a real, transition to a limit. Such an idealized transition is not something children are usually

\footnotetext{
${ }^{48}$ The 'predicative-impredcative' terminology goes back to Bertrand Russell's theory of ramified types.

${ }^{49}$ Being the tallest person in the room is an unproblematic impredicative concept, one might argue, because the number of people in the room is finite and can be mentally surveyed.

${ }^{50}$ See, e.g., Quine [1953], p. 127.

${ }^{51}$ See Cocchiarella [2007], chapter 4, $\$ 4$, for a description of this mechanism.

${ }^{52} \mathrm{This}$ is in fact Quine's position in [1953], p.127.
} 
capable of, but, as with the concept of a least upper bound, or of a converging sequence of numbers, it is based on a pattern of reflective abstraction that, with proper training and conceptual development, most of us come to acquire sometime in postadolescence. The same situation of a later development is also reflected in cultural history and the development of knowledge; that is, it was not until the 19th Century that the impredicative concepts of the differential calculus were finally given a clear mathematical and logical explication.

\section{Potential Infinity}

The number of concepts that the human mind can have formed as part of its conceptual repertoire at any given time, like the number of predicate expressions of a language, is finite. But aren't both concepts and linguistic expressions potentially infinite? That is, even though the number of concepts and the number of expressions of a language is always finite, nevertheless, at any given time, they both can be extended by the development of new concepts and expressions for those concepts.

Now, as already noted in the case of nominalism, there is a problem with how we explicate the notion of a potential, as opposed to an actual, infinity. Consider, for example, a type of entity $\mathbf{A}$. It would seem that we could say that there are potentially infinitely many $\mathbf{A}$ if the following claims are all true for all positive integers $n$ :

1. There is at least one $\mathbf{A}$.

2. There are at least two $\mathbf{A}$.

\section{$\vdots$}

n. There are at least $n$ many $\mathbf{A}$.

\section{:}

Now the problem is that this infinite set of sentences is true only if there are infinitely many $\mathbf{A}$, and not just potentially infinitely many. In other words, the above account fails as an account of potential, as opposed to actual, infinity.

Of course, in saying that the number of concepts, or expressions, is potentially, but not ever actually, infinite we mean not just the above but also that the number is always finite. So, what we need to add is the additional assumption that the number of $\mathbf{A}$ is not just potentially infinite but also finite. That is, let us consider adding the statement that there are only finitely many $\mathbf{A}$ to the above set of sentences as axiom $\mathbf{0}$ :

0. There are finitely many $\mathbf{A}$.

1. There is at least one $\mathbf{A}$. 
2. There are at least two $\mathbf{A}$.

$\vdots$

n. There are at least $n$ many $\mathbf{A}$.

$\vdots$

But is this set of sentences consistent? In fact, it is. Note that if the set were inconsistent, then a contradiction would be derivable in a finite number of steps, whereas of course any finite subset of this set is trivially consistent. ${ }^{53}$ From this it follows by the compactness of the syntactic derivability relation that the entire set is consistent, and therefore it has a second-order model. ${ }^{54}$ But, unfortunately, the model must be "nonstandard," in which case "finite' does not mean what it standardly means. In particular, there would then seem to be no possible world at which all of the sentences in the set are true in the standard sense.

Now the claim that there are potentially infinitely many concepts, or expressions, does not mean that there is some time, or even some possible world, when the mind has grasped all of the concepts that it can possibly form or construct, or that a language contains all of the expressions that could ever be added to it. That is, what we really mean is that for every natural number $n$ it is possible (in nature if not in time) that there are $n$ concepts, or expressions, even though it is necessary that the number of concepts, or expressions, in any physically possible world, or at any time, is finite. In other words, instead of the above set of sentences, what we mean by finite but potentially infinite is the following set of sentences (for all positive integers $n$ ):

\footnotetext{
${ }^{53}$ This result was first noted by Alfred Tarski many years ago.

${ }^{54}$ Finitude is not a first-order notion, and it is for that reason that the model must be second-order.
} 
0. Necessarily, there are finitely many A.

1. Possibly, there is at least one $\mathbf{A}$.

2. Possibly, there are at least two A.

$\vdots$

n. Possibly, there are at least $n$ many $\mathbf{A}$.

$\vdots$

Here, by possibility and necessity, we could mean nothing more than Diodorus's notion in which 'Possibly $S$ ' means that $S$ is or will be the case, and 'Necessarily $S$ ' means that $S$ is and always will be the case; or we could use Aristotle's notion where 'Possibly $S$ ' means that either $S$ was, is, or will be the case, and 'Necessarily $S$ ' means that $S$ is always the case. ${ }^{55}$ In other words, the sentences state in effect that for each natural number $n$ there is a time when we will have formed $n$ many concepts, or have introduced $n$ many expressions of a language, even though at no time will we have formed an infinity of concepts, or have introduced an infinity of expressions. On this reconstruction the entire set of sentences is not only consistent but also has a "standard" interpretation in which a standard model is associated with every moment of time (or world).$^{56}$ That interpretation, however, requires that there be an infinite amount time (assuming the quantization of time).

Of course, if we really do have an infinite number of parallel worlds in the multiverse, then we could just leave the meaning of possibility and necessity as a form of physical possibility and necessity, i.e., as being possible (or necessary) in some (every) parallel world. The point in any case is that potential infinity does make sense when modalized in this way.

\section{Conceptual Realism}

Conceptualism, if it is not to become a form of idealism, needs to be supplemented with an account of how our capacity for concept formation is related to the world, and in particular how referential and predicable concepts can be used to refer to and characterize things in the world. But then, because our cognitive abilities are causally grounded in our biology, the most natural framework for this purpose is some form of natural realism. In fact, as a formal ontology, natural realism in turn presupposes some form of conceptualism as a vehicle by which it can be expressed. We have described this combined framework elsewhere as a form of conceptual natural realism. ${ }^{57}$ The idea is that, depending on

\footnotetext{
${ }^{55}$ See Cocchiarella [2007], chapter 2, for a formalization of Diodorus's and Aristotle's notions of necessity and possibility in terms of tense logic.

${ }^{56}$ For a semantics of this see Cocchiarella [2007], chapter 2.

${ }^{57}$ See Cocchiarella [2007], chapter 12.
} 
our scientific theories and experimental data, natural properties and relations can be assumed to correspond to some, but of course not all, of our predicable concepts; and, similarly, natural kinds can be assumed to correspond to some, but not to all, of our common-name concepts, i.e., the classifying concepts that function as part of conceptualism's theory of reference. ${ }^{58}$ The combined framework, together with an S4 or S5 modal logic depending on how we understand the relation between the parallel worlds of the multiverse, results in a modal moderate realism that is a modern counterpart of Aristotelian essentialism.

In addition to natural realism, one can extend the framework of conceptualism by an intensional realism that amounts to a mitigated form of Platonism. This intensional realism comes about at a stage of conceptual development at which, through a pattern of reflective abstraction, we attempt to grasp the content of our predicable concepts as if they were objects. Syntactically, this pattern of reflective abstraction is represented by the process of nominalization, where, e.g., we transform predicate expressions such as 'is wise' and 'is triangular' into abstract singular terms such as 'wisdom' and 'triangularity'. These abstract singular terms will be vacuous, of course, unless we also assume a corresponding reality of intensional objects. That is just what is assumed in conceptual Platonism.

The Platonist assumption can be mitigated, however, by understanding intensional objects as somehow the product of a cultural evolution corresponding to the institutionalization in language of the process of nominalization. In other words, instead of assuming that intensional objects exist independently of the world, they are taken as not pre-existing the evolution of consciousness and culture but as following and depending upon that evolution as projections of our concepts on the level of objects. ${ }^{59}$ Such an assumption, however, would seem to limit the cardinality of intensional objects to a potential infinity. In any case, the combined framework of conceptualism, naturalism and intensional realism as a mitigated form of logical realism shows how one can reconcile both Platonism and Aristotelian essentialism in a coherent and unified formal ontology.

\section{$7 \quad$ Plural Objects}

As already noted, the proper and common names of conceptualism's theory of reference occur as parts of quantifier phrases, which in conceptualism represent the noun phrases of natural language. But just as predicate expressions can be nominalized and occur as abstract singular terms, so too proper and common names can be "nominalized" and allowed to occur as "objectual" terms, i.e., as arguments of predicates. Thus we distinguish the use of the common name 'man' in the referential (quantifier) phrase 'Every man' from its transformation into 'mankind', as when we say that Socrates is a member of mankind. We say

\footnotetext{
${ }^{58}$ See Cocchiarella [2007], chapter 12, for a development of conceptual natural realism and Aristotelian essentialism.

${ }^{59}$ For a fuller discusssion of this extension of conceptualism to conceptual intensional realism see Cocchiarella [2007], chapter $5, \S 3$.
} 
that 'mankind' is an "objectual" term rather than a singular term because what it denotes is not a single object but a group, namely, the group of humans taken collectively, which is a plural object, or plurality, as opposed to its members, each of which is a single individual object. This group is also what the plural of 'man', namely, 'men', denotes. Thus, we say not only that Socrates is a member of mankind but also that he is one among men. Taking groups as plural objects means that the notion of an object includes both plural objects and single objects, whereas the notion of an individual is by definition that of a single object.

Nominalized common names, as we have shown elsewhere, can be the basis of a logic of plurals within the framework of conceptual realism. ${ }^{60}$ Such a logic is needed to semantically account for irreducible forms of plural reference and predication. The well-know Geach sentence 'Some critics admire only each other', for example, which semantically says that there is a group of critics who admire only other members of the group, cannot be analyzed in first-order logic alone; and it would be ontologically misleading to analyze it in terms of set theory. Unlike a set, a group in the sense intended here is a plurality of individuals and not an abstract object. ${ }^{61}$

A logic of plurals is needed not just as a semantical framework for plural reference and predication in natural language and our commonsense framework, but, and perhaps more importantly, also for an ontological account of the properties of groups of objects in our scientific theories. The temperature and pressure of a volume of gas, for example, are really properties of the group of atoms or molecules in that volume rather than properties of the individual atoms or molecules that make it up. The visual, auditory, and other sensory properties of different modules of the brain are properties of the groups of neurons that make up those modules rather than of the individual neurons in the group. Similarly, the dispersion and redistribution of different populations of species of plants and animals are statistical properties of the groups of plants and animals and not of the individuals in those groups.

Plural objects are really what Bertrand Russell once called "classes as many" as opposed to "classes as one," i.e., as opposed to individual abstract entities such as sets. ${ }^{62}$ There are three main features of classes as many. The first is that, unlike sets, there is no empty class as many, which means that a name, proper or common, that names nothing has nothing as its extension. The second is that a class as many of one object is just that one object, which means that the extension of a name that names one thing is just that one thing. This shows that the extension of a proper name is just the object, if any, that the name denotes in its role as a singular term. The third feature is that, unlike sets, classes as many are plural objects, or pluralities, which means that they are

\footnotetext{
${ }^{60}$ See Cocchiarella [2007], chapter 11, for a development of the logic of plurals.

${ }^{61}$ For a variety of other examples of irreducible sentences that express irreducible plural references and/or predications see Cocchiarella [2007], chapter 11. It is noteworthy, incidentally, that Lésniewski's ontology, which is also called a logic of names, is reducible to the logic of classes as many. See Cocchiarella [2007], chapter 11, for the details of this reduction.

${ }^{62}$ See Russell [1903], chapter VI.
} 
literally made up of their members and cannot be construed as single entities the way sets are; and in particular it means that a class as many of more than one member, which is what we mean by a group, cannot "itself" be a member of any other class as many. Groups, in other words, have their being in their members in a more fundamental sense than sets do.

As indicated, there is no empty class as many. But what about the universal class? In set theory there is no universal class as one, i.e., there is no universal set as an abstract individual. But what about the universal class as many? The situation, it turns out, is rather complicated.

First, if nothing exist, which is allowed in our logic, then of course there is no universal class as many. Second, it is provable in the logic of classes as many that if something exists, then some individual, i.e., some single object, must exist. In other words plural objects, or groups, can exist only if there are single objects that are their members. This is quite unlike the situation in set theory where sets, or classes as one, exist whether or not there are any urelements, i.e., individuals that are not sets. In addition, it is provable in the logic of classes as many that if there are two individuals, i.e., two single objects, then the universal class as many does not exist. ${ }^{63}$ In other words, under the realistic assumption that there are many concrete, physical objects in the world - which of course are individuals or single objects - then there is no universal class as many.

But what if there is just one single object, one individual in the entire universe? That individual is of course identical with the class as many having just that one object as its only member, and other than that one class there are no other classes as many. But then isn't that class co-extensive with the universal class as many, and hence if that class exists - and it does because its only member does - doesn't the universal class then exist as well?

Significantly, such a conclusion follows only if we already know that the universal class exists. ${ }^{64}$ In other words, even if it were true that that one individual is the universal class, that is something we cannot know unless we already know that the universal class exists.

Finally, let us consider the situation when there are a countably infinite number of individuals, i.e., single objects, in the world. Does it then follow by Cantor's power-set theorem that there are an uncountably infinite number of groups of individuals, i.e., of classes as many of more than one member? And if so, wouldn't that show that there is more to the notion of a group than that of a simple plurality? That is, if out of $\aleph_{0}$ many individuals we can obtain $2^{\aleph_{0}}$ many groups of individuals, then doesn't that show that there is something abstract about groups? Perhaps. But curiously, Cantor's proof is not provable in the logic of classes as many when applied to the class of individuals, which suggest that there is nothing abstract about plural objects after all. Indeed, as

\footnotetext{
${ }^{63}$ For these details about theorems see Cocchiarella [2007], chapter 11.

${ }^{64}$ The universal class, were it to exist, would be the extension of the complex common name 'object that is self-identical'. Thus, to say that a class $A$ is co-extensive with the universal class means only that an object belongs to $A$ if, and only if, that object is self-identical. It does not follow in the free logic of classes as many that then the universal class exists if $A$ exists. For formal details, see Cocchiarella [2007], chapter 11, $§ 3$.
} 
the extensions of concepts that proper and common-name concepts stand for, it would be surprising if their cardinality were to exceed that of the concepts whose extensions they are. ${ }^{65}$

\section{References}

[1] Campbell, Keith, 1981, "The metaphysics of abstract particulars", Midwest Studies in Philosophy, vol. 6: 477-88.

[2] Cocchiarella, Nino B., 1987, Logical Studies in Early Philosophy, Ohio State University Press, Columbus.

[3] Cocchiarella, Nino B., 2007, Formal Ontology and Conceptual Realism, Springer, Synthese Library, vol. 339. Dordrecht.

[4] Dedekind, R. 1888, Was sind und was sollen dies Zahlen?, Braunschweig.

[5] De Witt, B. and N. Graham, eds., 1973, The Many-Worlds Interpretation of Quantum Mechanics, Princeton University Press, Princeton.

[6] Gatlin, Lila L., 1972, Information Theory and the Living System, Columbia University press, NY.

[7] Greene, Brian, 1999, The Elegant Universe, Random House Inc., NY.

[8] Greene, Brian, 2004, The Fabric of the Cosmos, Space, Time, and the Texture of Reality, Alfred A. Knopf, NY.

[9] Gribbin, John, 2001, Hyperspace, Our Final Frontier, DK Pub. Inc., NY.

[10] Henkin, Leon, 1962, "Nominalistic analysis of mathematical language," Logic, Methodology and Philosophy of Science, Proceedings of the 1960 International Congress, eds., Ernest Nagel, Patrick Suppes, and Alfred Tarski, Stanford University Press, Stanford, pp. 187-93.

[11] Luminet, Jean-Pierre, Glenn D. Starkman, and Jeffrey R. Weeks, "Is Space Finite?", Scientific American, April, 1999.

\footnotetext{
${ }^{65}$ Where the number of individuals is finite and greater than 1 , i.e., where there are $n$ many single objects in the universe, for some positive integer $n>1$, then, by a simple inductive argument it can be shown that the number of classes as many of objects, single and plural, is $2^{n}-1$, and hence that there are $2^{n}-(n+1)$ plural objects. Of course $2^{n}-1>n$, where $n>1$.

But where the number of single objects is $\aleph_{0}$, we cannot show that the number of classes as many is $2^{\aleph_{0}}-1$ (which is just $2^{\aleph_{0}}$ ). The attempt to derive a contradiction by Cantor's argument of assuming a 1-1 mapping $f$ of all of the classes as many of individuals into the class of all individuals fails because the Cantor diagonal class of individuals $x$ such that $x \notin f(x)$ must be known to exist (or equivalently have a member) in order to derive a contradiction. If it exists, then a contradiction follows, and what this shows is that the Cantor class as many, like the Russell class as many, doesn't exist in the logic of classes as many (which is "free" of existential presuppositions).
} 
[12] Penrose, Roger, 2004, The Road to Reality, A Complete Guide to the Laws of the Universe, Alfred A. Knopf, N.Y.

[13] Quine, Willard van Orman, 1953, From a Logical Point of View, Harvard University Press, Cambridge.

[14] McCall, Storrs, 1994, A Model of the Universe: Space-Time, Probability, and Decision, Clarendon Press, Oxford.

[15] Russell, Bertrand, 1903, The Principles of Mathematics, second edition (1938), Norton \& Co., NY.

[16] Shannon, Claude E. and Warren Weaver, 1949, The Mathematical Theory of Communication, University of Illinois Press, Urbana.

[17] Tegmark, Max, 2003, "Parallel Universes," in Science and Ultimate Reality: From Quantum to Cosmos, honoring John Wheeler's 90th birthday, J.D. Barrow, P.C.W. Davies, \& C.L. Harper, eds., Cambridge University Press, Cambridge.

[18] Tarski, Alfred, 1924, "Sur les ensembles finis," Fundamenta Mathematicae, vol. 6: 43-95.

[19] Williams, D.C., 1953, "The elements of being," The Review of Metaphysics, vol. 6 . 